\begin{tabular}{|c|c|c|}
\hline 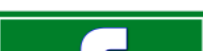 & Int.J.Curr.Microbiol.App.Sci (2021) 10(08): 156-163 & \\
\hline & $\begin{array}{l}\text { International Journal of Current Microbiology and Applied Sciences } \\
\text { ISSN: 2319-7706 Volume } \mathbf{1 0} \text { Number } 08 \mathbf{( 2 0 2 1 )} \\
\text { Journal homepage: } \underline{\text { http://www.ijcmas.com }}\end{array}$ & 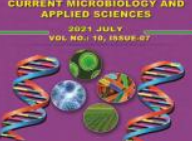 \\
\hline $\begin{array}{l}\text { EXCELLENT } \\
\text { PUBLISHERS }\end{array}$ & & \\
\hline
\end{tabular}

Original Research Article

https://doi.org/10.20546/ijcmas.2021.1008.020

\title{
Early and Late Onset Pre-eclampsia - Maternal and Perinatal Outcome in Tertiary Care Hospital
}

\author{
Lakshmi Kiran*, Geeta Sri and Ch. Sowjanya Lakshmi \\ Rangaraya Medical College, Kakinada, India \\ *Corresponding author
}

\section{A B S T R A C T}

Keywords

Pre-eclampsia, early onset pre-

eclampsia, late onset pre-

eclampsia, maternal outcome and perinatal outcome

Article Info

\section{Accepted:}

12 July 2021

Available Online:

10 August 2021
Pre-eclampsia is one of the major causes of morbidity and mortality for both mother and baby.(1) Pre-eclampsia occurs in 3-11\% of all pregnancies.(2) It is categorized into early onset and late onset according to the period of gestation. Pre-eclampsia that is diagnosed before 34 weeks of gestation is early onset and when diagnosed after 34 weeks of gestation is considered as late onset. To compare maternal and perinatal outcome in early onset (EOPE) and late onset preeclampsia (LOPE). This is a prospective observational study which included 177 pre-eclampsia patients, who were admitted in the Department of Obstetrics and Gynecology, Rangaraya Medical College at GGH, Kakinada, from SEP -2020 to FEB -2021 (6 months). Those who developed pre-eclampsia before 34 weeks of gestational age were identified and taken as early onset pre-eclampsia. those who developed after 34 weeks gestational age were identified as late onset preeclampsia. Maternal and perinatal outcome was compared between those groups. Incidence of pre-eclampsia is 3.3\%, of which early onset (EOPE) is $1.3 \%$ and late onset pre-eclampsia $2 \%$. Compared with late onset pre-eclampsia (LOPE), early onset pre-eclampsia (EOPE) group has higher rates of eclampsia $(43.4 \%$ vs $27.7 \%)$, abruption placenta (14.4\% vs $12 \%)$, pulmonary edema (1.4\% vs $0 \%)$ and there was no intergroup difference in the composite maternal outcome. A significantly higher number of women with early onset pre-eclampsia (EOPE) developed severe features during the disease course $(73.9 \%$ vs $60 \%)$ and most of them required treatment with antihypertensive drugs like labetalol (86.4\% vs 62.9 $\%$ ), nifedipine (13\% vs 37\%) (P value 0.005). Babies born to mother with early onset pre-eclampsia (EOPE) had significantly higher rate of NICU admissions $(50.7 \%$ vs $37 \%)$ and ventilator support (37.6\% vs $25 \%)$. IUDs $(20.2 \%$ vs $8.3 \%)$ are significantly higher in number in early onset pre-eclampsia (EOPE). The study shows that women with early onset pre-eclampsia (EOPE) had more adverse maternal and perinatal outcomes than those with late onset pre-eclampsia (LOPE). But the difference was not statistically significant. 


\section{Introduction}

Pre-eclampsia is a hypertensive disorder specific to pregnancy.(2) Over the past decades, the incidence of pre-eclampsia has increased in some regions. It complicates up to $3-11 \%$ of all pregnancies and is associated with serious maternal and fetal outcome. Hence early detection and management of the disease is necessary to decrease its global burden.

According to new terminology, pre-eclampsia without evidence of end organ damage is termed as pre-eclampsia without severe features. However, the presence of end organ damage defines severe pre-eclampsia.(3)

Pre-eclampsia is defined as a multisystem disorder of unknown aetiology characterized by development of hypertension to the extent of 140/90 $\mathrm{mm}$ of $\mathrm{Hg}$ or more with proteinuria after the 20th week in a previously normotensive and non-proteinuria woman. Eclampsia is characterized by woman with pre-eclampsia complicated with grand-mal seizures and/or coma. Superimposed preeclampsia is characterized by occurrence of new onset of proteinuria in woman with chronic hypertension. HELLP syndrome is one of the complications of pre-eclampsia characterized by Haemolysis (H) Elevated Liver enzymes (EL) Low Platelet count (LP).

The basic underlying pathology is endothelial dysfunction and intense vasospasm. Normally at 10 to 12 weeks endovascular trophoblast invades up to decidual segments called first wave. At 16 to 18 weeks trophoblast invades up to the myometrial segments, so that the spiral arterioles become distended, tortuous, and funnel shaped called second wave. This change transforms the spiral arterioles into low resistance, low pressure, and high flow systems. In pre-eclampsia there is failure of the second wave leading to reduction of blood supply to the feto-placental unit. Hypertension diagnostic criteria is rise of blood pressure at least $140 \mathrm{~mm} \mathrm{Hg}$ if the previous blood pressure not known or a rise in systolic pressure at least $30 \mathrm{~mm} \mathrm{Hg}$ or a rise in diastolic blood pressure of at least $15 \mathrm{~mm} \mathrm{Hg}$ over the previously known blood pressure or rise of $20 \mathrm{~mm} \mathrm{Hg}$ mean arterial blood pressure (MAP) over the previous reading or MAP $>=105 \mathrm{~mm} \mathrm{Hg}$ should be consider as significant. Presence of total proteins in $24 \mathrm{hr}$ urine of $0.3 \mathrm{~g}$ or more or urine protein creatinine ratio more than 0.3 or equal to $1+$ (persistent $30 \mathrm{mg} / \mathrm{dl}$ ) protein in at least 2 random clean catch urine samples tested 4 hours apart in the absence of urinary tract infection is considered as significant.(3)

In recent years, a new disease classification is given based on the timing of onset which has gained attention.(4) It consists of early onset pre-eclampsia occurring before 34 weeks of gestation and late onset pre-eclampsia occurring at or after 34 weeks of gestation. The diagnostic criteria are the same for EOPE and LOPE, in fact this simple division has better prognostic implications than mild vs severe terminology.(4)

This is a prospective study which examined the effects of EOPE and LOPE on composite maternal and perinatal outcome. Clinical and laboratory findings, maternal and perinatal outcomes were compared between groups.

\section{Materials and Methods}

The study is a type of prospective observational study and was performed in the Department of Obstetrics and Gynecology in RMC, GGH, Kakinada, Andhra Pradesh from SEP 2020- FEB 2021. 177 pre-eclampsia patients which attributes to $3.3 \%$ of 5360 patients who were delivered at our hospital were included in study. Early onset preeclampsia group consists of 69 women who were diagnosed before 34 weeks of gestational 
age. Late onset pre-eclampsia group consists of 108 women who were diagnosed after 34 weeks of gestational age. The two groups were compared according to age, BMI, parity, past $\mathrm{H} / \mathrm{O}$ pre-eclampsia, GDM, clinical findings, maternal medication with antihypertensive therapy (labetalol, nifedipine), $\mathrm{MgSO} 4$ therapy. Induction of labor and mode of delivery (LSCS, NVD) were compared. Investigation parameters like umbilical artery doppler, hemoglobin percentage, platelet count, coagulation profile, LFT, RFT were compared. Maternal complications like eclampsia, complete and partial HELLP, abruption placenta, AKI, PPH were compared. Perinatal outcomes including birth weight, small for GA, still birth, live birth, IUD, neonatal death, 5 min Apgar, NICU admission and ventilator support were compared.

\section{Exclusion Criteria}

Pregnant women with essential hypertension before 20 weeks of GA, chronic hypertension, preexisting renal disease.

\section{Statistical Analysis}

Data is expressed as $\mathrm{n}(\%)$ and mean with standard deviation. Student $t$ test was used to compare the 2 groups depending on normality of data. Proportions were compared using chi square test depending on applicability for the two groups. The statistical tests were 2 side D and performed at a significance level $\mathrm{p}=0.05$. The analysis was conducted using SPSS statistics.

\section{Results and Discussion}

Total number of 177 women at more than 20 weeks of GA with pre-eclampsia were taken in this study, divided into early onset preeclampsia and late onset pre-eclampsia groups ( $n=69$ and 108 respectively). The mean age at diagnosis in EOPE group is 23.3 years,
Standard deviation is 1.86 and in LOPE mean is 27.69 years and standard deviation 3.04. The mean age at diagnosis was 30 weeks 36 weeks in EOPE and LOPE, respectively. The mean age at admission in EOPE was 32.6 weeks and S.D is 0.69 , average duration of pre-eclampsia onset to delivery was 12 days. In LOPE group the mean age at admission was 37.05 weeks and S.D is 1.11 , mean gestational age at delivery was 37 weeks. Average duration of onset of pre-eclampsia to delivery was 5.2 days. The results were similar with the study conducted by Pooja Wadhwani et al., at pgimer, Chandigarh i.e., A study to compare maternal and perinatal outcome in early vs late onset pre-eclampsia.(5)

Hematological and biochemical parameter are summarized in table 2. The mean s. creatinine value in EOPE and LOPE group were $0.64 \pm 0.24 \mathrm{mg} / \mathrm{dl}$ and $0.55 \pm 0.43$ respectively, the results were similar with study done by Pooja Wadhwani et al., at pgimer, Chandigarh i.e. A study to compare maternal and perinatal outcome in early vs late onset preeclampsia.(5)

A significantly higher number of women with EOPE than those with LOPE developed severe features during their disease course, required treatment with anti-hypertensive therapy ( $\mathrm{p}<$ 0.005). The rate of induction was little bit higher in EOPE group than those with LOPE group (57.9 \% vs 50.9\%). However, the cesarean section rate was significantly higher in EOPE than LOPE. The results were similar with study "Conducted by Gomathy, E et al., at sri Devaraj urs medical college, Kolar, Karnataka.(6)

A Total of - 60 women (86\%) with EOPE developed adverse outcomes vs 80 women (70\%) with LOPE. The maternal complications were little bit higher in EOPE than LOPE. The results were similar with the study "Maternal morbidity associated with 
early onset and late onset pre-eclampsia "conducted by SarkaLisonkova from university of British Columbia.(7)

Pulmonary edema, abruption placenta, AKI were observed more in EOPE group. The results were similar with study "early onset and late onset preclampsia: a comprehensive cohort study of laboratory and clinical findings according to the new isshp criteria "conducted by Anna wojtowiczetal from jagiellonian university medical college.

The women with EOPE had higher number of IUGR (52\% vs $46 \%)$ babies. Umbilical artery changes were similar in both groups. The rate of intra uterine deaths (IUD) were higher in EOPE group than LOPE group i.e., $20.2 \%$ vs $8.3 \%$. Also, babies born to EOPE women had significantly higher number of NICU admissions (50.7\% vs 37\%) and ventilator support ( $37.6 \%$ vs $25 \%)$ and this is similar to the results of the study comparison of clinical and perinatal outcome in early and late onset pre-eclampsia conducted by Riza Madazli et $a l$. , from Istanbul university.(4)

The primary strength of our study is that it was prospective study and performed in tertiary care hospital with a relevant number of study subjects.

The distinction between EOPE and LOPE is a relatively modern concept and is becoming widely accepted as a better indicator of disease significance. Approaching as an early onset and late onset pre-eclampsia gives us better idea about understanding of complex etiopathogenesis of this medical enigma. There are limited number of studies and reviews that have compared characteristics of EOPE and LOPE.(6) In study conducted by sankalisnkova that shows maternal and perinatal morbidity and mortality is higher in EOPE group.(7) This study demonstrates that
EOPE poses a high risk of maternal and perinatal outcome(4). The results were similar with the study conducted by Pooja Wadhwani et al., at pgimer, Chandigarh i.e., A study to compare maternal and perinatal outcome in early vs late onset pre-eclampsia.(5)

The incidence of pre-eclampsia is $3.3 \%$. Primi gravida, family history of hypertension or pre-eclampsia, new paternity, placental abnormalities, obesity, thrombophilia, preexisting vascular diseases are some of the risk factors. Pre-eclampsia occurring at early gestational age presents clinicians a challenge of needing to balance the risk of perinatal death and severe neonatal morbidity.(8) The exact cause of pre-eclampsia is unknown, but maternal, placental factors are considered to be involved in etiology of the disease. It has been suggested that EOPE is more strongly associated with internal placental factors, whereas the LOPE onset may be primarily due to predisposing maternal factors (5).

It is worth mentioning that patients in the studied population were predominantly primi which is known to be a major risk factor (8).

Clinical classification of pre-eclampsia (nonsevere, severe) is arbitrary and principally dependent on the level of blood pressure for management purposes. This is same for EOPE and LOPE. Severe pre-eclampsia is characterized by a persistent systolic blood pressure above or equal to $160 \mathrm{~mm} \mathrm{Hg}$ or diastolic blood pressure above $110 \mathrm{~mm} \mathrm{Hg}$, Presence of proteinuria, Presence of oliguria (< 400ml for 24 hours), Platelet count less than 100000/mm3, Elevated liver enzymes, Cerebral or visual disturbances, Persistent severe epigastric pain, Retinal haemorrhages, papilledema, Pulmonary oedema, Foetal growth restriction, Serum creatinine more than $1.1 \mathrm{mg} / \mathrm{dl}$. 
Table.1 Summarizes the base line demographic details if women with EOPE AND LOPE.

\begin{tabular}{|c|c|c|c|}
\hline Characteristic & Early Onset PE 69 & $\begin{array}{c}\text { Late Onset PE } \\
\mathbf{1 0 8}\end{array}$ & P Value \\
\hline Age & 20-27(mean: $23.3 ;$ s.d: 1.86$)$ & $\begin{array}{c}20-35(\text { mean: } \\
27.69 ; \text { s.d: } 3.04)\end{array}$ & \\
\hline BMI & $22-29$ & $22-30$ & \\
\hline Primi & $44(63 \%)$ & $62(57 \%)$ & 0.4 \\
\hline Multi & $25-36.2$ & $46(42.5 \%)$ & 0.4 \\
\hline GDM & $05(7.2 \%)$ & $21(19.4 \%)$ & 0.9 \\
\hline $\begin{array}{c}\text { Past h/o of Pre- } \\
\text { eclampsia }\end{array}$ & $07(10.1 \%)$ & $140-160$ & 0.9 \\
\hline $\begin{array}{c}\text { Systolic bp At } \\
\text { admission }\end{array}$ & $140-160$ & $80-120$ & 0.9 \\
\hline $\begin{array}{c}\text { Diastolic bp at } \\
\text { admission }\end{array}$ & $80-120$ & & 0.9 \\
\hline
\end{tabular}

Table.2

\begin{tabular}{|c|c|c|}
\hline Variable & Early onset PE & Late onset PE \\
\hline Hemoglobin & $10.9 \pm 1.06$ & $9.7 \pm 1.09$ \\
\hline Platelet count & $1,80,000 \pm 50,000$ & $1,60,000 \pm 65,000$ \\
\hline Urea & $30 \pm 12.5$ & $29 \pm 10.6$ \\
\hline Sr. Creatinine & $0.64 \pm 0.24$ & $0.55 \pm 0.43$ \\
\hline Sr. Bilirubin & $0.8 \pm 2.3$ & $0.7 \pm 0.2$ \\
\hline SGOT & $46 \pm 45.4$ & $42 \pm 30.7$ \\
\hline SGPT & $45 \pm 46.2$ & $44 \pm 38.7$ \\
\hline ALP & $230 \pm 150.8$ & $250 \pm 98.3$ \\
\hline
\end{tabular}

Table.3 Disease severity correlation, labor, delivery details in early onset and late onset pre-eclampsia

\begin{tabular}{|c|c|c|c|}
\hline Treatment & $\begin{array}{c}\text { Early onset PE } \\
\text { (total = 69) }\end{array}$ & $\begin{array}{c}\text { Late onset PE } \\
\text { (total = 108) }\end{array}$ & P value \\
\hline Antihypertensive therapy & $\begin{array}{c}60-86.9 \% \\
09-13 . \%\end{array}$ & $\begin{array}{c}68-62.9 \% \\
40-37 \% \\
30-27 \%\end{array}$ & 0.005 \\
\hline $\begin{array}{c}\text { a) labetalol } \\
\text { b) nifedipine }\end{array}$ & 09 & $65-60 \%$ & \\
c) both & $51-73.9 \%$ & $84-77 \%$ & 0.08 \\
\hline Pe with severe features & $40-57.9 \%$ & $55-50.9 \%$ & 0.08 \\
\hline Mgso4 THERAPY & $40-57.9 \%$ & $43-39.8 \%$ & 0.7 \\
\hline Induction of labor & $36-52 \%$ & & 0.7 \\
\hline Cesarean section & & & \\
\hline
\end{tabular}


Table.4 Maternal complications in women with early onset and late onset pre-eclampsia

\begin{tabular}{|c|c|c|c|}
\hline Complication & Early onset PE 69 & Late onset PE108 & P value \\
\hline Eclampsia & $3043.4 \%$ & $3027.7 \%$ & 0.1 \\
\hline Complete hellp & $45.7 \%$ & $04-3.7 \%$ & 0.3 \\
\hline Partial hellp & $34.3 \%$ & $10-9.2 \%$ & 0.3 \\
\hline Abruption & $10-14.4 \%$ & $13-12 \%$ & 0.3 \\
\hline Pulmonary edema & $01-1.4 \%$ & 0 & 0.7 \\
\hline AKI & $01-1.4 \%$ & $01-0.9 \%$ & 0.7 \\
\hline PPH & $11-15.9 \%$ & $22-20.3 \%$ & 0.7 \\
\hline CVA & 0 & 0 & \\
\hline Retinal detachment & 0 & 0 & \\
\hline
\end{tabular}

Table.5 Perinatal outcome in women with early onset and late onset pre-eclampsia

\begin{tabular}{|c|c|c|c|}
\hline Parameter & Early Onset PE & Late Onset PE & P Value \\
\hline Small for GA & $26-37.6 \%$ & $37-34.2 \%$ & 0.3 \\
\hline $\begin{array}{c}\text { Umbilical artery } \\
\text { Doppler changes }\end{array}$ & $20-28.9 \%$ & $31-28.7 \%$ & 0.3 \\
\hline Still birth & $01-1.4 \%$ & $02-1.8 \%$ & 0.9 \\
\hline Live birth & $54-78.2 \%$ & $97-89.8 \%$ & 0.9 \\
\hline Birth weight & $36-52.1 \%$ & $50-46.2 \%$ & 0.4 \\
\hline $2.5 \mathrm{~kg}$ & $33-47.8 \%$ & $59-54.6 \%$ & \\
\hline$>2.5 \mathrm{~kg}$ & $18-26 \%$ & $30-27.7 \%$ & 0.8 \\
\hline 5 min APGAR score & $35-50.7 \%$ & $40-37 \%$ & 0.3 \\
\hline NICU admission & $26-37.6 \%$ & $27-25 \%$ & 0.3 \\
\hline Ventilator support & $11-15.9 \%$ & $19-17.5 \%$ & 0.08 \\
\hline Neonatal death & $14-20.2 \%$ & $9-8.3 \%$ & 0.08 \\
\hline IUD & & & \\
\hline
\end{tabular}

The study conducted by Fang et al., did not find any difference in risk factors between early onset and late onset pre-eclampsia. (9) According to ISSHP study maternal age has no significance in outcome. Mother with EOPE than those with LOPE had adverse outcomes, but the difference did not reach statistical significance. (7) Mean age of EOPE group was 23 years and LOPE group was 28 years. (7)

The most common maternal complications were HELLP syndrome, abruption placenta and eclampsia in both groups. No significant inter group difference was noted in maternal outcomes $(4,6)$ Interestingly pulmonary edema and AKI cases were observed in both groups. The major complications of pre-eclampsia during pregnancy includes eclampsia, accidental haemorrhage, renal failure, eye complications, preterm labour, HELLP syndrome, cerebral haemorrhage, acute respiratory distress syndrome. During labour, eclampsia, post-partum haemorrhage are the major complications. During puerperium, shock, sepsis, DIC (Disseminated Intravascular Coagulation), eclampsia are the complications. Foetal complications include foetal growth restriction, prematurity, asphyxia, intra uterine death. Remote 
complications may present as residual hypertension, recurrent pre-eclampsia, chronic renal disease.(3)

The neonatal death rate was higher in EOPE group than in the LOPE group, although that was not statistically significant. (4) Adverse neonatal complications were significantly more in babies born to mothers with EOPE i.e., NICU admissions (50.7\% vs $37 \%$ ), ventilator support $(37.6 \%$ vs $25 \%)$. The result is consistent with those of most previous relevant study (5) Gestational age at birth was the most important factor contributing to neonatal outcomes. The greater of adverse perinatal outcome in EOPE group might have been due to early prematurity itself than the severity of pre-eclampsia (9)

As it is a tertiary care hospital base study, we recruited many number of pre-eclampsia cases. In our study we had EOPE (68) and LOPE (108) according to the measurements of BP. Antihypertensive treatment was given immediately to both EOPE and LOPE groups. In this study there were no maternal mortality.

In summary EOPE differed from LOPE mainly in terms of adverse maternal and fetoplacental conditions and severe complications

A high number of women with EOPE than those with LOPE developed severe features during their disease course and most of them required treatment with antihypertensive therapy. Perinatal morbidity rates were higher mother with EOPE than in those with LOPE.

Our findings indicate that women with EOPE had more adverse outcomes than those with LOPE, but the difference was not statistically significant. Prophylactic measures for prevention of pre-eclampsia are regular antenatal check-ups, daily intake of low dose aspirin, low molecular weight heparin in woman with thrombophilia and high-risk pregnancy, calcium supplementation $2 \mathrm{~g}$ per day, antioxidants, vitamin $\mathrm{C}$, vitamin $\mathrm{E}$, magnesium, zinc, fish oil, balanced diet rich in protein may play a significant role in preventing pre-eclampsia.

If persistent symptoms of severe preeclampsia, pulmonary oedema, hypoxia $(\mathrm{Pa}$ O2 < 95\%), hepatocellular injury (increase AST, ALT twice the normal), oliguria, abnormal coagulation profile, foetal growth restriction with non-reassuring foetal status, eclampsia, uterine artery doppler with reverse end diastolic flow (REDF) characters if present immediate delivery must be done.

Early detection of pre-eclampsia followed by immediate management with antihypertensive therapy along with clinical and laboratory findings definitely decrease maternal and perinatal morbidity and mortality.

\section{References}

1. Arias practice guide to high-risk pregnancy and delivery - chapter hypertensive disorders in pregnancy $4^{\text {th }}$ edition, pp.185-232

2. Williams Obstetrics hypertensive disorders in pregnancy chapter $28^{\text {th }}$ edition, pp.710-754

3. D C Dutta $9^{\text {th }}$ edition textbook of Obstetrics -Hypertensive disorders in pregnancy - chapter 18, pp.207-224

4. Comparison of clinical and perinatal outcomes in early and late onset preeclampsia

5. Riza madazli- Mehmet Aytac YukselMetehan Imamoglu, Abdullah Tuten, Muhmut Oncul, Burcu Aydin, Gokhan Demirayak. Springer- verlag berlin Heidelberg 2014

6. A study to compare maternal and perinatal outcome in early vs late onset 
pre-eclampsia.

7. Pooja wadhwani, pradipkumarsaha, jasinderkaurkalra, shalinGainder, venkataseshansundaram. obstetGynecol 2020; 270-277

8. Early onset and late onset pre-eclampsia maternal and perinatal outcome in a rural teritiary health care.

9. Gomathy. E, Lahari Akurati, Kondareddy Radhika international journal of reproduction, contraception, obstetrics and gynecology 2018JUN; 2266-2269

10. Maternal morbidity associated with early onset and late onset pre-eclampsia. sankalisnkova, Yasser sabr. Chantal mayer, Carmen young, Amanda skoll, k.s. joseph. American college of obstetrics and gynecology vol 124

11. Early and late onset of $\mathrm{PE}-\mathrm{a}$ comprehensive cohort study of laboratory and clinical findings to the view of ISHHP criteria - vol. 2019

12. Risk factors of early onset and late onset pre-eclampsia.

Adisorn Aksornphusitaphong and Vorapong Phupong. The journal of obstetrics and gynecology vol.39; 627-63

\section{How to cite this article:}

Lakshmi Kiran, Geeta Sri and Sowjanya Lakshmi. 2021. Early and Late Onset Pre-eclampsia Maternal and Perinatal Outcome in Tertiary Care Hospital. Int.J.Curr.Microbiol.App.Sci. 10(08): 156-163. doi: https://doi.org/10.20546/ijcmas.2021.1008.020 